CASE REPORT

\title{
Large cell neuroendocrine carcinoma of the ampulla of Vater with glandular differentiation
}

\author{
S-P Cheng, T-L Yang, K-M Chang, C-L Liv
}

J Clin Pathol 2004;57:1098-1100. doi: 10.1136/icp.2004.019414

Large cell neuroendocrine carcinoma of the ampulla of Vater is extremely rare. A 55 year old woman presented with an ampullary tumour causing pancreaticobiliary obstruction and a pancreaticoduodenectomy was performed. Microscopically, the tumour was diagnosed as a CD117 positive large cell neuroendocrine carcinoma with glandular differentiation. Four months later the patient developed a general recurrence. The metastatic tumours showed CD117 negativity and pure neuroendocrine features. The patient died of disease six months after diagnosis. It is postulated that the two components originated from a common multipotential stem cell. The clinical behaviour of ampullary large cell neuroendocrine carcinomas appears to be highly aggressive, with early metastases and a fatal outcome.

$\mathrm{P}$ rimary neuroendocrine tumours of the ampulla of Vater are rare. These tumours, similar to those of the respiratory tract and remainder of the gastrointestinal tract, present a histological spectrum of increasing malignancy from low grade carcinoids to high grade small cell carcinoma. ${ }^{1}$ Large cell neuroendocrine carcinoma (LCNEC) is a distinctive clinicopathological entity that is associated with aggressive clinical behaviour, with only one well documented ampullary LCNEC having been reported. ${ }^{2}$ Here, we present a case of LCNEC of the ampulla of Vater that contained an admixed minor glandular component. The case also draws attention towards the occurrence of dual differentiation in relation to KIT overexpression.

\section{CASE REPORT}

A 55 year old woman presented with a two month history of dull epigastric pain. Her past medical history was unremarkable. An abdominal ultrasonography revealed dilatation of the biliary tree with no stones visible. An endoscopic retrograde cholangiopancreatography showed an ulcerated polypoidal lesion in the ampullary region. Biopsies of this mass were consistent with poorly differentiated carcinoma with neuroendocrine features. A computed tomographic scan of the abdomen demonstrated a small enhancing ampullary mass associated with enlarged peripancreatic lymph nodes. The liver was normal except for the biliary duct dilatation. An angiomyolipoma $1.5 \mathrm{~cm}$ in diameter was also noted in the right kidney. The patient underwent radical pancreaticoduodenectomy including systematic nodal dissection. Her postoperative recovery was uneventful. The patient declined further chemotherapy or radiotherapy.

Four months later the patient manifested intestinal obstruction. Abdominal computed tomography demonstrated multiple nodules of various sizes in both lobes of the liver, suggestive of hepatic metastases. Prolonged ileus with poor response to chemotherapy required reoperation. At laparotomy, metastatic dissemination to the liver, mesentery, and peritoneum was found. Palliative gastrojejunostomy was carried out to bypass the site of obstruction. The patient died of disease six months after initial diagnosis. No necropsy was performed.

\section{PATHOLOGY}

Pathological examination of the pancreaticoduodenectomy specimen revealed an ulcerated, firm, grey/white mass, measuring $1.8 \mathrm{~cm}$ in greatest dimension, located in the ampulla of Vater. The ampullary opening was circumferentially obstructed by tumour. Routine haematoxylin and eosin stained sections through the ampulla of Vater revealed multiple nests of tumour cells that showed a prominent organoid pattern of growth, formed rosettes, and invaded the muscular wall of the ampulla (fig lA). The tumour nests did not frankly extend into the duodenal subserosa. The tumour cells were large and had a moderate amount of eosinophilic cytoplasm, a low nuclear to cytoplasmic ratio, and round or oval nuclei of varying sizes. The nuclei were vesicular and contained coarsely granular chromatin with frequent nucleoli. Mitotic figures and atypical mitoses were frequently seen (>50 mitotic figures per 10 high power fields). The tumour contained multiple foci of necrosis. Lymphovascular invasion was identified whereas perineural invasion was absent. Pancreatic tissue and surgical margins were unremarkable.

Immunohistochemical studies were performed using the avidin-biotin-peroxidase complex technique with commercially available primary antibodies. Neoplastic cells were positive for chromogranin and synaptophysin, but were negative for neurone specific enolase and carcinoembryonic antigen (fig 1B). Ki-67 immunostaining revealed a proliferation index of $60 \%$. In addition, the tumour cells showed strong immunoreactivity for CD117 (polyclonal; 1/50 dilution; Dako, Carpinteria, California, USA) (fig lC).

Approximately $5 \%$ of the tumour was composed of foci showing vague glandular differentiation (fig $1 \mathrm{D}, \mathrm{E}$ ). These tumour cells were cuboidal to low columnar in shape, with apical snouting, and lined half of the well formed glandular lumen. They looked like normal well differentiated adenocarcinoma cells but with only mild nuclear atypia. Areas showing an intermingled admixture with a transitional zone, in which the cells shared features common to both elements, were identified. No apparent mucin positivity was seen in the lumens. Histochemical staining for periodic acid Schiff with diastase showed weak staining for intracytoplasmic mucin (fig 1F). The glandular components were consistently negative for neuroendocrine markers. Two of 23 regional lymph nodes contained metastases. These metastatic deposits had microscopic features similar to those of the primary ampullary tumour, including the presence of glandular differentiation (fig lG). The neuroendocrine cells were the major component with extensive infarct-like necrosis.

Abbreviations: LCNEC, large cell neuroendocrine carcinoma 

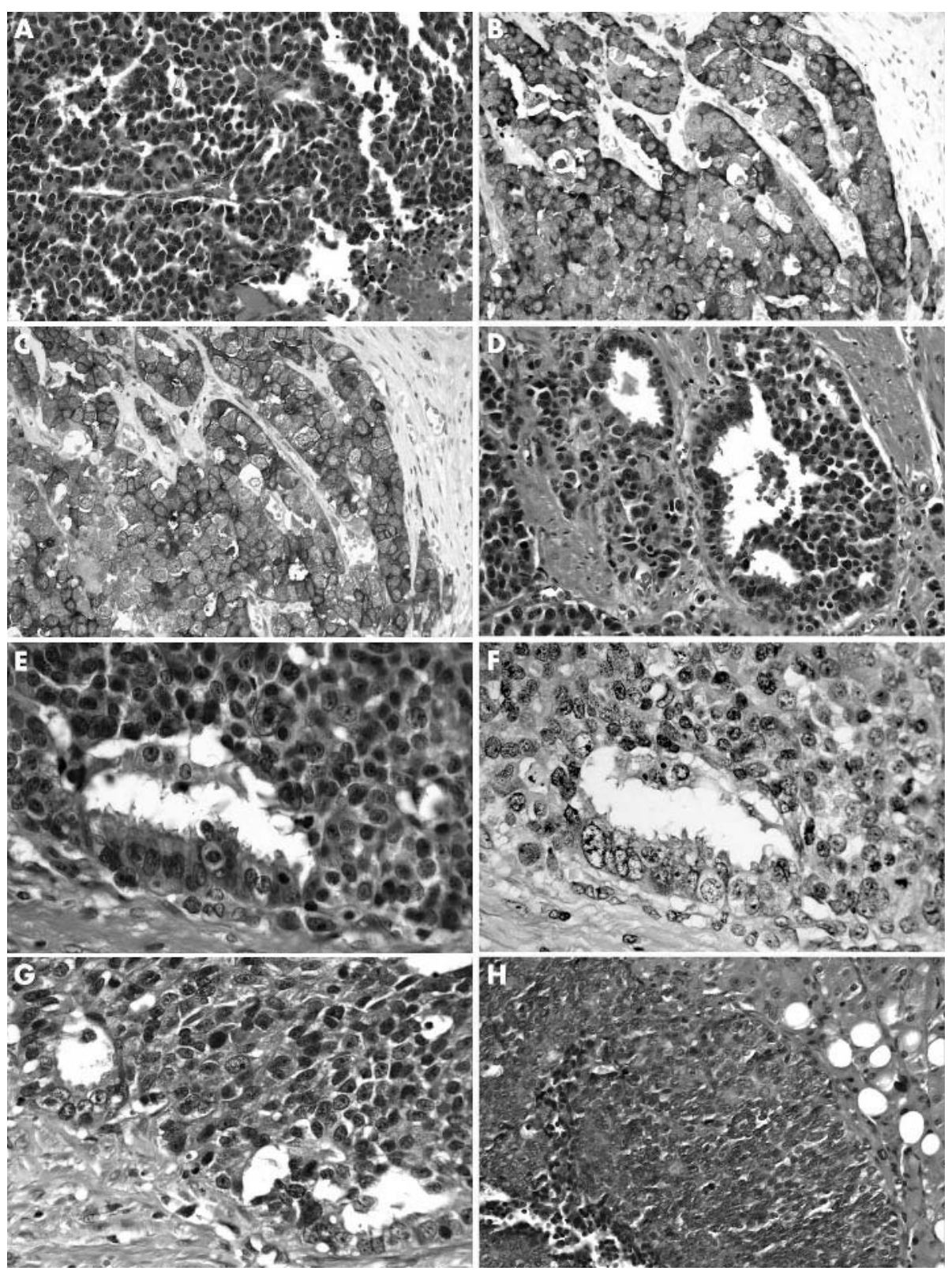

Figure 1 Large cell neuroendocrine carcinoma. (A) The tumour cells are arranged in an organoid growth pattern, showing peripheral nuclear palisading, rosette-like structures, and many mitotic figures. A focus of necrosis is present. (B) Tumour cells show strong immunoreactivity for chromogranin. (C)

Immunohistochemical staining for $C D 117$ shows diffuse positivity. (D, E) Histological features of dual differentiation with an intermingling
transitional area between the glandular and neuroendocrine components. (F) Some tumour cells of the glandular component are periodic acid Schiff positive with diastase resistance. No apparent intraluminal mucin is seen. (G) Area of glandular differentiation in the metastatic foci of regional lymph nodes. (H) Microscopic examination of the metastatic liver fumour shows neuroendocrine carcinoma without a glandular component.
The biopsy specimen taken from the hepatic and peritoneal metastases five months after the pancreaticoduodenectomy showed only large cell neuroendocrine carcinoma (fig 1H). A component with glandular differentiation was not present. Staining for CD117 was negative.

\section{DISCUSSION}

Malignancy arising at the ampulla of Vater is not uncommon and adenocarcinoma accounts for most of these malignancies. Neuroendocrine tumours of the ampulla of Vater have been reported, mostly in small series. ${ }^{1}$ Travis and colleagues proposed a three grade scheme for pulmonary neuroendocrine tumours with substantial reproducibility, including low grade typical carcinoid, intermediate grade atypical carcinoid, and high grade categories of LCNEC and small cell carcinoma. ${ }^{3}$ Extrapulmonary LCNEC is a highly aggressive neoplasm resembling its pulmonary counterpart both histologically and in its highly malignant behaviour. ${ }^{2}$ Our case is unique because glandular differentiation in addition to the typical light microscopic and immunohisto- chemical features of ampullary LCNEC has not been reported previously.

Neuroendocrine tumours with a mixed histological pattern have been reported in pulmonary and extrapulmonary locations..$^{4-6}$ These neoplasms have been referred to in various terms as combined or mixed tumours. Adenocarcinoid tumours, which histologically contain both ductal and neuroendocrine differentiation, most commonly occur in the appendix. ${ }^{4}$ Five isolated reports of adenocarcinoid tumours of the periampullary region have been described in the literature. ${ }^{5}$ However, none of the carcinoid components in previous reports met the diagnostic criteria of LCNEC. We believe that the absence of immunoperoxidase staining for carcinoembryonic antigen and the lack of mucin production in our case mean that this neoplasm should be classified as a LCNEC with glandular differentiation. Ruffini and associates analysed 1158 resected lung tumours and found that 19 (1.6\%) had a combined small cell carcinoma/LCNEC plus a non-neuroendocrine carcinoma. ${ }^{6}$ They showed that patients in the combined group have a poor prognosis and that 
Take home messages

- We present a rare case of large cell neuroendocrine carcinoma of the ampulla of Vater with glandular differentiation

- Divergent differentiation was also present in the metastatic lymph nodes but was absent after relapse, along with the loss of CD1 17 overexpression

- Large cell neuroendocrine carcinomas appear to be highly aggressive tumours, with early metastases and a fatal outcome

survival rates are similar to those seen in pure small cell carcinoma. It appears that ampullary LCNEC follows a similar pattern. As in our case, despite the presence of glandular differentiation, LCNEC of the ampulla of Vater is a high grade malignancy with a dismal prognosis.

"Glandular differentiation in addition to the typical light microscopic and immunohistochemical features of ampullary large cell neuroendocrine carcinoma has not been reported previously."

The intimate arrangement between the two differentiated cell types raises a question of the histogenesis of these tumours. Results from a molecular study suggest that both the carcinoid and glandular components of a mixed tumour share a common multipotential stem cell origin. ${ }^{7}$ It is noteworthy that Ruffini et al reported that the metastatic lymph nodes showed pure histology, rather than the mixed tumour type. ${ }^{6}$ In our present patient, the two components coexisted in the metastatic foci as an intricate admixture with a transition zone. There are three possible mechanisms of metastasis, namely: (1) the common precursors undergo metastasis to the lymph nodes, where the progenitor tumour cells multiply and differentiate as the primary tumour; (2) both cell types of the primary tumour possess the ability to metastasise; and (3) tumour cells of the glandular component are passively carried with the LCNEC cells to the vascular bed of metastatic sites. The common origin theory is more compatible with the intermixing of the two components within the primary tumour and metastases.

Recently, it was shown that KIT was overexpressed in 55$60 \%$ of pulmonary LCNEC tumour cells. ${ }^{89}$ This protein, the product of the c-kit protooncogene, is a transmembrane tyrosine kinase receptor that regulates the normal develop- ment of different cell lines and also promotes tumour cell proliferation and blocks apoptosis in some haemopoietic disorders and solid malignancies. Loss of CDI17 overexpression after chemotherapy is often seen in small cell lung carcinoma. ${ }^{9}$ One possible explanation is that chemotherapy induces the selection of CD117 negative neoplastic clones in relapsing disease. Our case showed loss of both CD117 overexpression and glandular differentiation in the metastatic lesions after chemotherapy. It remains unclear whether this is a cause and effect association, or whether it arises from the selected tumour cells having an undifferentiated pattern and more aggressive behaviour.

In conclusion, we present a case of LCNEC of the ampulla of Vater with glandular differentiation. Divergent differentiation was also present in the metastatic lymph nodes but was absent after relapse, along with the loss of CD117 overexpression.

\section{Authors' affiliations}

S-P Cheng, T-L Yang, C-L Liu, Department of Surgery, Mackay Memorial Hospital, Taipei 10449, Taiwan

K-M Chang, Department of Pathology, Mackay Memorial Hospital

Correspondence to: Dr T-L Yang, Department of Surgery, Mackay Memorial Hospital, 92, Sec 2, Chung-Shan North Road, Taipei 10449, Taiwan; yangtl@yam.com

Accepted for publication 8 May 2004

\section{REFERENCES}

1 Emory RE Jr, Emory TS, Goellner JR, et al. Neuroendocrine ampullary tumors: spectrum of disease including the first report of a neuroendocrine carcinoma of non-small cell type. Surgery 1994;115:762-6.

2 Cavazza A, Gallo M, Valcavi R, et al. Large cell neuroendocrine carcinoma of the ampulla of Vater. Arch Pathol Lab Med 2003;127:221-3.

3 Travis WD, Rush W, Flieder DB, et al. Survival analysis of 200 pulmonary neuroendocrine tumors with clarification of criteria for atypical carcinoid and its separation from typical carcinoid. Am J Surg Pathol 1998;22:934-44.

4 Levendoglu H, Cox CA, Nadimpalli V. Composite (adenocarcinoid) tumors of the gastrointestinal tract. Dig Dis Sci 1990;35:519-25.

5 Alex WR, Auerbach HE, Pezzi CM. Adenocarcinoid tumor of the ampulla of Vater. Am Surg 1998;64:355-9.

6 Ruffini E, Rena O, Oliaro A, et al. Lung tumors with mixed histologic pattern. Clinico-pathologic characteristics and prognostic significance. Eur J Cardiothorac Surg 2002;22:701-7.

7 Vortmeyer AO, Lubensky IA, Merino MJ, et al. Concordance of genetic alterations in poorly differentiated colorectal neuroendocrine carcinomas and associated adenocarcinomas. J Natl Cancer Inst 1997;89:1448-53.

8 Araki K, Ishii G, Yokose T, et al. Frequent overexpression of the c-kit protein in large cell neuroendocrine carcinoma of the lung. Lung Cancer 2003;40:173-80.

9 Rossi G, Cavazza A, Marchioni A, et al. Kit expression in small cell carcinomas of the lung: effects of chemotherapy. Mod Pathol 2003; 16:1041-7. 